\title{
Subgroup Analysis by Liver Metastasis in the FRESCO Trial Comparing Fruquintinib versus Placebo Plus Best Supportive Care in Chinese Patients with Metastatic Colorectal Cancer
}

Shukui Qin (D)', Rui-Hua $X u^{2}$, Lin Shen $\left(D^{3}\right.$, Jianming $\mathrm{Xu}^{4}$, Yuxian $\mathrm{Bai}^{5}$, Lei Yang (ID ${ }^{6}$, Yanhong Deng ${ }^{7}$,

Zhen-dong Chen ${ }^{8}$, Haijun Zhong ${ }^{9}$, Hongming Pan ${ }^{10}$, Weijian Guo' Yongqian Shu ${ }^{12}$, Ying Yuan ${ }^{13}$, Jianfeng Zhou ${ }^{14}$, Nong $X u^{15}$, Tianshu Liu (iD) ${ }^{16}$, Dong $\mathrm{Ma}^{17}$, Changping $\mathrm{Wu}^{18}$, Ying Cheng ${ }^{19}$, Donghui Chen ${ }^{20}$, Wei $\mathrm{Li}^{21}$, Sanyuan Sun (D2) ${ }^{22}$, Zhuang $\mathrm{Yu}^{23}$, Peiguo Cao (iD) ${ }^{24}$, Haihui Chen ${ }^{25}$, Jiejun Wang ${ }^{26}$, Shubin Wang ${ }^{27}$, Hongbing Wang $^{28}$, Ning Wang ${ }^{29}$, Bin Zhang ${ }^{29}$, Qiang Zhang ${ }^{29}$, Weiguo $\mathrm{Su}^{30}$, Xiaojun $\mathrm{Guo}^{30}$, Jin $\mathrm{Li}^{31}$

'Cancer Center, Jinling Hospital, Nanjing, People's Republic of China; ${ }^{2}$ Department of Medical Oncology, Sun Yat-sen University Cancer Center, State Key Laboratory of Oncology in South China, Guangzhou, People's Republic of China; ${ }^{3}$ Department of Gastrointestinal Oncology, Key Laboratory of Carcinogenesis and Translational Research (Ministry of Education), Peking University Cancer Hospital \& Institute, Beijing, People's Republic of China; ${ }^{4}$ Department of Medical Oncology, The Fifth Medical Center of Chinese PLA General Hospital, Beijing, People's Republic of China; ${ }^{5}$ Department of Medical Oncology, Harbin Medical University Cancer Hospital, Harbin, People's Republic of China; ${ }^{6}$ Department of Medical Oncology, Nantong Cancer Hospital, Nantong, People's Republic of China; ${ }^{7}$ Department of Medical Oncology, The Sixth Affiliated Hospital of Sun Yat-sen University, Guangzhou, People's Republic of China; ${ }^{8}$ Department of Medical Oncology, The Second Hospital of Anhui Medical University, Hefei, People's Republic of China; ${ }^{9}$ Department of Medical Oncology, Zhejiang Cancer Hospital, Hangzhou, People's Republic of China;

${ }^{10}$ Department of Medical Oncology, Sir Run Run Shaw Hospital, College of Medicine, Zhejiang University, Hangzhou, People's Republic of China; "'Department of Medical Oncology, Shanghai Medical College, Fudan University Shanghai Cancer Center, Shanghai, People's Republic of China; ${ }^{12}$ Department of
Objective: The aim of the present subgroup analysis of the FRESCO trial is to determine the efficacy and hepatotoxicity of fruquintinib in Chinese patients with metastatic CRC with liver metastasis (CRLM) who were receiving third-line or posterior-line therapy.

Methods: Overall survival (OS) and progression-free survival (PFS) were evaluated by Kaplan-Meier method. Hazard ratio (HR) was estimated through Cox proportional hazards model. Hepatotoxicity was coded using the standardized MedDRA queries of hepatic failure, fibrosis, cirrhosis, and other liver injury-related conditions and graded using the Common Terminology Criteria Adverse Events grades. The efficacy of fruquintinib in patients with CRLM was evaluated in various subgroups.

Results: A total of 287 (69.0\%) patients with metastatic CRC had liver metastasis (LM, fruquintinib: 185 and placebo: 102). Median OS in patients with CRLM was significantly prolonged with fruquintinib compared with placebo (8.61 months vs 5.98 months; $\mathrm{HR}=0.59,95 \% \mathrm{CI}, 0.45-0.77$, $\mathrm{P}<0.001)$. In patients with CRLM, the incremental median PFS for patients in the fruquintinibtreated group was significantly higher than in the placebo group (median PFS: 3.71 vs.1.84 months; $\mathrm{HR}=0.22,95 \%$ CI: $0.17-0.30 ; \mathrm{P}<0.001)$. Compared with placebo, significant improvements in OS were observed with fruquintinib in LM patients regardless of lung metastasis, prior target therapy, and K-RAS status. In patients with CRLM, treatment-emergent hepatotoxicities of any grade occurred in 7 (3.8\%) patients in the fruquintinib group vs $2(2.0 \%)$ in the placebo group.

Conclusion: Fruquintinib demonstrated a statistically significant increase in OS and PFS as compared with placebo in Chinese patients with CRLM. The hepatotoxicity of fruquintinib was less reported, and comparable with placebo in patients with CRLM.

ClinicalTrials.gov Identifier: NCT02314819.

Keywords: colorectal cancer, FRESCO trial, fruquintinib, liver metastasis

\section{Plain Language Summary}

- First subgroup analysis of the efficacy and hepatic safety data of fruquintinib in Chinese patients with mCRC with liver metastasis (CRLM) who were receiving third-line therapy.

- Fruquintinib significantly lowered the death and recurrence risks for CRLM patients, with median OS and median PFS 8.61 months and 3.71 months, respectively. Of note, the incidence of treatment-emergent hepatotoxicity was similar in CRLM patients compared to the placebo group.

- Survival benefits from fruquintinib were consistent across subgroups regardless of prior anti-VEGF/EGFR treatment, primary tumor site or K-RAS status in patients with CRLM in FRESCO. 
Medical Oncology, Jiangsu Provincial Hospital, Nanjing, People's Republic of China;

${ }^{13}$ Department of Medical Oncology, The Second Affiliated Hospital of Zhejiang University School of Medicine, Hangzhou, People's Republic of China; ${ }^{14}$ Department of Medical Oncology, Peking Union Medical College Hospital, Beijing, People's Republic of China; ${ }^{15}$ Department of Medical Oncology, The First Affiliated Hospital of Zhejiang University College of Medicine, Hangzhou, People's Republic of China; ${ }^{16}$ Department of Medical Oncology, Fudan University Zhongshan Hospital, Shanghai Medical College, Shanghai, People's Republic of China; ${ }^{17}$ Department of Medical Oncology, Guangdong Provincial People's Hospital, Guangzhou, People's Republic of China; ${ }^{18}$ Department of Medical Oncology, The First People's Hospital of Changzhou, Changzhou, People's Republic of China; ${ }^{19}$ Department of Medical Oncology, Jilin Province Cancer Hospital, Changchun, People's Republic of China; ${ }^{20}$ Department of Medical Oncology, Shanghai Jiaotong University Affiliated First People's Hospital, Shanghai, People's Republic of China; ${ }^{21}$ Department of Medical Oncology, The First Hospital of Jilin University, Changchun, People's Republic of China; ${ }^{22}$ Department of Medical Oncology, Xuzhou Central Hospital, Xuzhou, People's Republic of China; ${ }^{23}$ Department of Medical Oncology, The Affiliated Hospital of Medical College Qingdao University, Qingdao, People's Republic of China; ${ }^{24}$ Department of Medical Oncology, The Third Xiangya Hospital of Central South University, Changsha, People's Republic of China; ${ }^{25}$ Department of Medical Oncology, Liuzhou Worker's Hospital, Liuzhou, People's Republic of China; ${ }^{26}$ Department of Medical Oncology, Shanghai Changzheng Hospital, The Second Military Medical University, Shanghai, People's Republic of China; ${ }^{27}$ Department of Medical Oncology, Peking University Shenzhen Hospital, Beijing University, Shenzhen, People's Republic of China; ${ }^{28}$ Department of Medical Oncology, The Affiliated Hospital of Xuzhou Medical College, Xuzhou Medical College, Xuzhou, People's Republic of China; ${ }^{29}$ Eli Lilly and Company, Shanghai, People's Republic of China;

${ }^{30}$ Hutchison MediPharma Limited, Shanghai, People's Republic of China; ${ }^{31}$ Department of Medical Oncology, Tongji University Shanghai East Hospital, Shanghai, People's Republic of China

Correspondence: Jin $\mathrm{Li}$ Department of Medical Oncology, Tongji University Shanghai East Hospital, Shanghai, People's Republic of China

Tel +86I376I222III

Email lijin@csco.org.cn

\section{Introduction}

Colorectal cancer (CRC) is the most commonly diagnosed cancer and the fourth leading cause of cancer mortality in the world. ${ }^{1,2}$ In China, approximately 376000 new cases of CRC per year were reported in 2015 and have continued to grow. ${ }^{3}$ A large-scale, realworld study in China indicated that liver is one of the most common metastatic sites in Chinese patients with advanced $\mathrm{CRC},{ }^{3,4}$ with approximately $70 \%$ of patients having liver metastasis (LM) at the beginning of third-line therapy. ${ }^{5,6}$ In real-world evidence, among 6764 Chinese patients with Tumor, Node, Metastasis (TNM) classification records at diagnosis, about $44 \%$ presented with TNM Stage IV at diagnosis; among them, 52\% had $\mathrm{LM}^{7}$ Liver metastasis is one of the major causes of death in patients with $\mathrm{CRC}^{8}$. The disease impairs liver function either from replacement of normal parenchyma, restriction of vascular inflow (particularly portal venous inflow), or obstruction of biliary drainage and thus significantly enhances CRC progression. ${ }^{8}$

Surgical resection has become the standard curative treatment for patients with resectable colorectal liver metastasis (CRLM) ${ }^{8,9}$ Unfortunately, only about $20 \%$ of patients with CRLM have resectable cancer. ${ }^{10}$ More than $70 \%$ of patients with CRLM after liver resection develop recurrence in the remnant liver. ${ }^{9,10}$ Therefore, surgical resection for resectable CRLM is still a controversial and evolving topic within the realm of surgical oncology. ${ }^{8-10}$ FOLFOX (oxaliplatin, fluorouracil, and leucovorin) and FOLFIRI (irinotecan, leucovorin, and fluorouracil) are the 2 standard chemotherapy regimens (first-line therapy) for unresectable CRLM; $; 11,12$ however, they are associated with various histological patterns of hepatotoxicity, such steatohepatitis, sinusoidal obstruction syndrome, and nodular regenerative hyperplasia. ${ }^{13}$ Chemotherapy-associated with hepatotoxicity may negatively impact the ability to offer potentially curative therapy or increase morbidity in some patients. ${ }^{13,14}$ The accumulated injuries to the liver caused by chemotherapeutic agents during previous systemic treatment could affect the efficacy of chemotherapy. ${ }^{13-15}$ The combination of FOLFOX or FOLFIRI along with second-line therapies - namely anti-epidermal growth factor receptor (EGFR) therapies using agents such as cetuximab and panitumumab, as well as anti-vascular endothelial growth factor (VEGF) monoclonal antibody therapy with agents like bevacizumab-have been proven to increase the response rate and tumor shrinkage. ${ }^{8,12,16}$ However, most patients with metastatic CRC developed disease progression even after receiving systemic therapies in the first- or second-line settings. ${ }^{17,18}$ Therefore, there is a great need, and promise, for treatment options in the third-line setting for metastatic CRC, especially in China.

Fruquintinib is a potent, highly selective small-molecule inhibitor of VEGFR-1, -2 , and -3 that has been approved by China's National Medical Products Administration in 2018 to treat patients with metastatic CRC who had failed at least 2 prior standard antitumor therapies, including fluoropyrimidine, oxaliplatin, and irinotecan, with or without prior use of anti- VEGFR or anti-EGFR treatment. ${ }^{19}$ In the Phase 3 FRESCO trial, fruquintinib demonstrated a statistically significant and clinically meaningful overall survival (OS) benefit in Chinese patients with metastatic CRC. ${ }^{20}$ Fruquintinib also showed an acceptable safety and tolerability profile in Chinese patients with metastatic CRC. ${ }^{20}$

No data on efficacy and hepatotoxicity profile of fruquintinib in Chinese patients with CRLM who failed at least 2 prior standard antitumor therapies, including fluoropyrimidine, oxaliplatin, and irinotecan, with or without prior useof anti-VEGF or anti-EGFR treatment were available. Thus the aim of the present subgroup analysis of the FRESCO trial is to determine the efficacy and hepatotoxicity of fruquitinib in Chinese patients with CRLM who were receiving third-line or posterior-line therapies. 


\section{Materials and Methods}

Study Design and Patients

FRESCO is a multicenter, randomized, double-blind, placebo-controlled, phase 3 trial comparing fruquintinib versus placebo plus best supportive care (BSC) in Chinese patients with metastatic CRC (NCT02314819). ${ }^{20}$ The purpose of the present post hoc analysis of the FRESCO trial was to evaluate survival benefit of fruquintinib in patients with CRLM. In the present post hoc analysis of the FRESCO trial, the survival benefit of fruquintinib in Chinese patients with metastatic CRC with and without LM was assessed. The patients with and without LM were separated in this subgroup analysis. Also, hepatotoxicity of fruquintinib in Chinese patients with metastatic CRC was assessed.

In the FRESCO trial, patients aged between 18 and 75 years with a histological and/or cytological diagnosis of metastatic CRC who had failed at least 2 prior treatments with fluoropyrimidine, oxaliplatin, and irinotecan were enrolled. Additional criteria included an Eastern Cooperative Oncology Group (ECOG) performance status of 0 or 1; a life expectancy of at least 3 months; and adequate bone marrow, liver, and renal function at baseline. Patients who had previously received any VEGFR inhibitors (regorafenib, ramucirumab, apatinib, axitinib, famitinib, or other tyrosine kinase inhibitors) or had other uncontrolled medical disorders were excluded. Patients who were previously treated with anti-EGFR or VEGF agents were eligible. Written informed consent was obtained from each patient and institutional ethics committee approval was obtained from each study center. The FRESCO trial was conducted in accordance with the laws and regulations in China regarding patient protection.

\section{Study Treatment}

In the FRESCO trial, eligible patients were randomized to receive fruquintinib plus $\mathrm{BSC}$ or placebo plus $\mathrm{BSC}$ in a 2:1 ratio. Patients in the fruquintinib group received fruquintinib $5 \mathrm{mg}$ once daily for 3 weeks on and 1 week off (28-day cycles). All randomized patients received fruquintinib plus BSC or placebo plus BSC until discontinuation due to intolerable toxicity, tumor progression, patient or investigator decision to stop treatment, or death.

\section{Efficacy Assessments}

The present subgroup analysis compared the OS and progression-free survival (PFS) after fruquintinib and placebo treatment along with BSC in Chinese patients with metastatic CRC with and without LM. Also, further subgroup analysis evaluating impact of clinicopathological factors on the efficacy of fruquintinib in Chinese patients with CRLM was evaluated in the following variables: lung metastasis status (yes and no); prior therapy status (with and without Anti-VEGF/Anti-EGFR); K-ras status (wildtype and mutant); primary disease sites (colon and rectal); primary tumor location (left and right); peritoneum metastasis status (yes and no); and time from metastasis to randomization ( $<18$ months and $\geq 18$ months). Moreover, best overall response (complete response, partial response, stable disease, and progressive disease), objective response rate (ORR, confirmed complete or partial response), disease control rate (DCR, complete or partial response, or stable disease recorded 8 weeks post randomization), and duration of stable disease were also assessed in Chinese patients with metastatic CRC with and without LM.

\section{Safety Assessments}

In the FRESCO trial, adverse events were assessed and graded during the study period, according to the National Cancer Institute Common Terminology Criteria for Adverse Events (CTCAE) version 4.03. Safety endpoints included treatment-emergent hepatotoxicity (by CTCAE grades and laboratory abnormalities). Hepatotoxicity was coded using the standardized MedDRA queries of hepatic failure, fibrosis, cirrhosis, and other liver injury-related conditions are graded using the CTCAE. Hy's law laboratory criteria were met if AST/ALT was $>3 \times \mathrm{ULN}$, total bilirubin was $>2 \times \mathrm{ULN}$, and alkaline phosphatase was $<2 \times$ ULN.

\section{Statistical Analysis}

For each subgroup, OS and PFS were evaluated by Kaplan-Meier method, and an unstratified Log rank test was used to compare treatment effect. Hazard ratio (HR) and its corresponding 95\% CI were estimated through Cox proportional hazards model, and $P$-value was generated from the Log rank test. Comparison related to categorical variables such as ORR and DCR was analyzed using a Fisher's exact test. Hepatotoxicity and hepatic laboratory abnormalities data were presented as frequencies and proportions. The significance level was set at a 2-sided $P=0.05$ for all statistical tests. All the statistical analyses were carried out using SAS version 9.2. 
Table I Demographic and Baseline Disease Characteristics mCRC by Liver Metastasis in FRESCO (Randomized Population)

\begin{tabular}{|c|c|c|c|c|}
\hline \multirow[t]{2}{*}{ Variables } & \multicolumn{2}{|c|}{ Patients with Liver Metastasis } & \multicolumn{2}{|c|}{ Patients without Liver Metastasis } \\
\hline & $\begin{array}{l}\text { Fruquintinib+BSC } \\
\qquad(\mathrm{N}=185)\end{array}$ & $\begin{array}{l}\text { Placebo+BSC } \\
(N=102)\end{array}$ & $\begin{array}{c}\text { Fruquintinib+BSC } \\
\qquad(\mathrm{N}=93)\end{array}$ & $\begin{array}{l}\text { Placebo+BSC } \\
\qquad(N=36)\end{array}$ \\
\hline \multicolumn{5}{|l|}{ Age group, $\mathbf{n}(\%)$} \\
\hline$<65$ years & $148(80.0)$ & $83(81.4)$ & $80(86.0)$ & $27(75.0)$ \\
\hline$\geq 65$ years & $37(20.0)$ & $19(18.6)$ & $13(14.0)$ & $9(25.0)$ \\
\hline \multicolumn{5}{|l|}{ Gender, n (\%) } \\
\hline Male/female & $109(58.9) / 76(41.1)$ & $74(72.5) / 28(27.5)$ & $49(52.7) / 44(47.3)$ & $23(63.9) / 13(36.1)$ \\
\hline \multicolumn{5}{|c|}{ ECOG performance status, $n$ (\%) } \\
\hline 0 & $53(28.6)$ & $31(30.4)$ & $24(25.8)$ & $6(16.7)$ \\
\hline I & $132(7 \mid .4)$ & $71(69.6)$ & $69(74.2)$ & $30(83.3)$ \\
\hline \multicolumn{5}{|c|}{ Primary disease site at first diagnosis } \\
\hline Colon & $108(58.4)$ & $55(53.9)$ & $39(41.9)$ & $15(41.7)$ \\
\hline Rectal & $72(38.9)$ & $40(39.2)$ & $53(57.0)$ & $20(55.6)$ \\
\hline Colon-rectal & $5(2.7)$ & $6(5.9)$ & $\mathrm{I}(\mathrm{I} . \mathrm{I})$ & $\mathrm{I}(2.8)$ \\
\hline Missing & 0 & $I(1.0)$ & 0 & 0 \\
\hline \multicolumn{5}{|c|}{ Primary tumor location at the time of diagnosis, $n$ (\%) } \\
\hline Left* & $137(74.1)$ & $85(83.3)$ & $77(82.8)$ & $30(83.3)$ \\
\hline Right** & $42(22.7)$ & $15(14.7)$ & $14(15.1)$ & $6(16.7)$ \\
\hline Both left and right & $4(2.2)$ & 0 & 0 & 0 \\
\hline Missing/Unknown & $2(1.1)$ & $2(2.0)$ & $2(2.2)$ & 0 \\
\hline \multicolumn{5}{|c|}{ Metastatic site, $\mathbf{n}(\%)$} \\
\hline Single & $7(3.8)$ & $3(2.9)$ & $6(6.5)$ & $\mathrm{I}(2.8)$ \\
\hline Multiple & $178(96.2)$ & $99(97.1)$ & $87(93.5)$ & $35(97.2)$ \\
\hline \multicolumn{5}{|c|}{ Stage of disease at the time of diagnosis, $n$ (\%) } \\
\hline 1 & $4(2.2)$ & $4(3.9)$ & $4(4.3)$ & 0 \\
\hline II & $22(11.9)$ & $8(7.8)$ & $12(12.9)$ & $10(27.8)$ \\
\hline III & $65(35.1)$ & $35(34.3)$ & $53(57.0)$ & $16(44.4)$ \\
\hline IV & $93(50.3)$ & $53(52.0)$ & $24(25.8)$ & $10(27.8)$ \\
\hline Missing & $\mathrm{I}(0.5)$ & $2(2.0)$ & 0 & 0 \\
\hline \multicolumn{5}{|c|}{$\begin{array}{l}\text { Time from first metastasis diagnosis to } \\
\text { randomization (months) }\end{array}$} \\
\hline Mean (SD) & $18.15(12.2)$ & $18.18(11.9)$ & $20.46(14.3)$ & $27.34(19.2)$ \\
\hline Median (min, max) & $15.18(2.1,61.6)$ & $14.74(1.9,63.6)$ & $17.68(0.9,79.0)$ & $23.03(4.0,81.6)$ \\
\hline \multicolumn{5}{|c|}{ Prior use of VEGF inhibitors, n (\%) } \\
\hline Yes & $53(28.6)$ & $27(26.5)$ & $31(33.3)$ & $13(36.1)$ \\
\hline \multicolumn{5}{|c|}{ Prior use of EGFR inhibitors, $n$ (\%) } \\
\hline Yes & $32(17.3)$ & $16(15.7)$ & $8(8.6)$ & $3(8.3)$ \\
\hline \multicolumn{5}{|c|}{ K-RAS gene status, n (\%) } \\
\hline Wild type & III (60.0) & $57(55.9)$ & $46(49.5)$ & $17(47.2)$ \\
\hline Mutant type & $74(40.0)$ & $45(44.1)$ & $47(50.5)$ & $19(52.8)$ \\
\hline \multicolumn{5}{|c|}{$\begin{array}{l}\text { Prior treatment lines on or above metastatic } \\
\text { disease, } \mathbf{n}(\%)\end{array}$} \\
\hline$\leq 3$ & $149(80.5)$ & $80(78.4)$ & $72(77.4)$ & $27(75.0)$ \\
\hline$>3$ & $36(19.5)$ & $22(21.6)$ & $21(22.6)$ & $9(25.0)$ \\
\hline
\end{tabular}

Notes: *Left region includes splenic flexure, descending, transverse, sigmoid colon, and rectum. **Right region includes cecum, ascending colon, and hepatic flexure. Abbreviations: BSC, best supportive care; ECOG, Eastern Cooperative Oncology Group; EGFR, epidermal growth factor receptor; KRAS, Kirsten rat sarcoma viral oncogene homolog; max, maximum; min, minimum; $\mathrm{N}$, total number patients; $n$, number of patients in each category; SD, standard deviation; VEGF, vascular endothelial growth factor. 


\section{A Patients With Liver Metastasis}

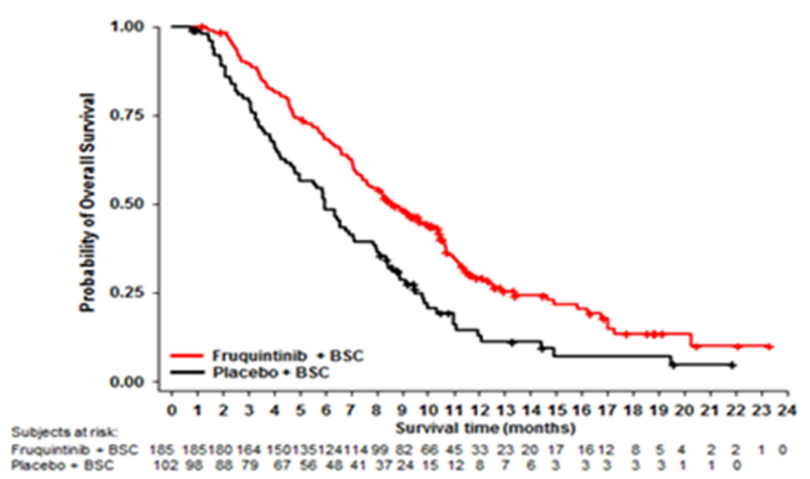

\section{B Patients Without Liver Metastasis}

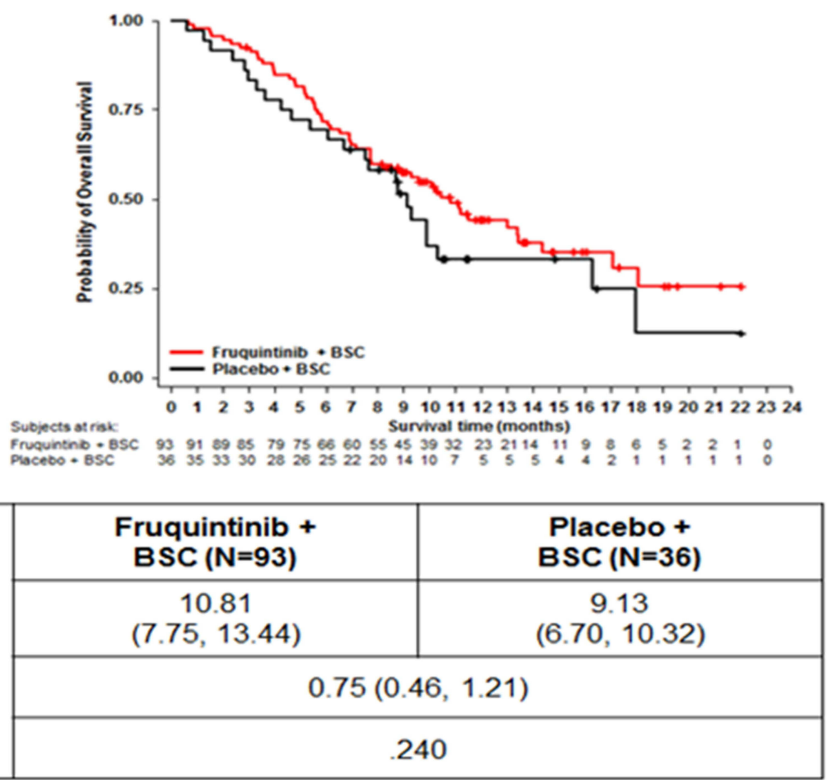

Figure I Comparison of overall survival in $m C R C$ patients with liver metastasis (A) and without liver metastasis (B). Abbreviations: BSC, best supportive care; HR, hazard ratio; $N$, total number patients.

\section{Results}

\section{Patient Disposition and Characteristics}

In the FRESCO trial, a total of 519 patients were screened, and 416 patients were randomized to receive fruquintinib $(\mathrm{n}=278)$ or placebo $(\mathrm{n}=138)$ between December 2014 and May 2016. Of these, 415 patients were included in the safety population (fruquintinib, $\mathrm{n}=278$; placebo, $\mathrm{n}=137$ ). A total of $287(69.2 \%)$ patients with mCRC had LM, including 185 in the fruquintinib-treated group and 102 in the placebo-treated group. The demographic and baseline disease characteristics of all randomized patients by LM are shown in Table 1.

\section{Efficacy}

Significant improvements in OS (Figure 1) and PFS (Figure 2) were found amongst the fruquintinib-treated mCRC patients with LM. In patients with LM, treatment with fruquintinib significantly prolonged the median OS compared to placebo (median OS: 8.61 vs 5.98 months; $\mathrm{HR}=0.59,95 \%$ CI: $0.45-0.77 ; \quad P<0.001)$. Also, in patients with CRLM, the incremental median PFS for patients in the fruquintinib group was significantly higher than in the placebo group (median PFS: 3.71 vs. 1.84 months; $\quad \mathrm{HR}=0.22, \quad 95 \% \quad \mathrm{CI}: \quad 0.17-0.30$;
$P<0.001)$. Furthermore, fruquintinib showed a significant greater response rate over placebo in patients with CRLM (ORR: $4.9 \%$ vs $0 \%, P=0.029$ and DCR; $62.2 \%$ vs $8.8 \%, P<0.001$ ) (Table 2 ).

Comparisons of OS and PFS for subgroups of CRLM patients are described in Figures 3 and 4, respectively. For metastatic CRC patients with LM, OS and PFS were significantly improved by fruquintinib compared to placebo, regardless of prior targeted therapy had been used or the time since diagnosis of first metastasis. Compared to placebo, significantly greater improvements in OS and PFS were observed in fruquintinib-treated LM patients with lung metastasis, K-RAS wild-type, left-sided primary tumors, without peritoneum metastasis, primary tumor site of rectal, or ECOG performance status (Figures 3 and 4).

\section{Safety}

In patients with liver metastasis, treatment-emergent hepatotoxicity of any grade occurred in 7 (3.8\%) patients in the fruquintinib group versus $2(2.0 \%)$ in the placebo group. Treatment-emergent hepatotoxicity events by patients with and without LM are summarized in Table 3. Most hepatotoxicities in patients treated with fruquintinib were grade $1(5 / 278=1.8 \%)$ or 


\section{A Patients With Liver Metastasis}

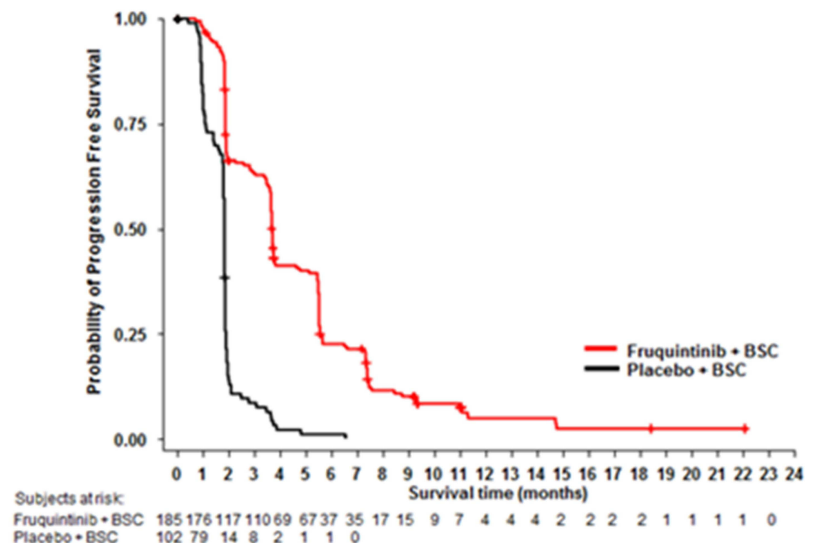

Fruquintnib*BSC
Placeoo $B S C$
102

\begin{tabular}{|c|c|c|}
\hline & $\begin{array}{c}\text { Fruquintinib + } \\
\mathrm{BSC}(\mathrm{N}=185)\end{array}$ & $\begin{array}{c}\text { Placebo + } \\
\text { BSC }(N=102)\end{array}$ \\
\hline $\begin{array}{l}\text { Median, months } \\
(95 \% \mathrm{Cl})\end{array}$ & $\begin{array}{c}3.71 \\
(3.65,3.81)\end{array}$ & $\begin{array}{c}1.84 \\
(1.81,1.84)\end{array}$ \\
\hline $\operatorname{HR}(95 \% \mathrm{CI})$ & \multicolumn{2}{|c|}{$0.22(0.17,0.30)$} \\
\hline
\end{tabular}

\section{B Patients Without Liver Metastasis}

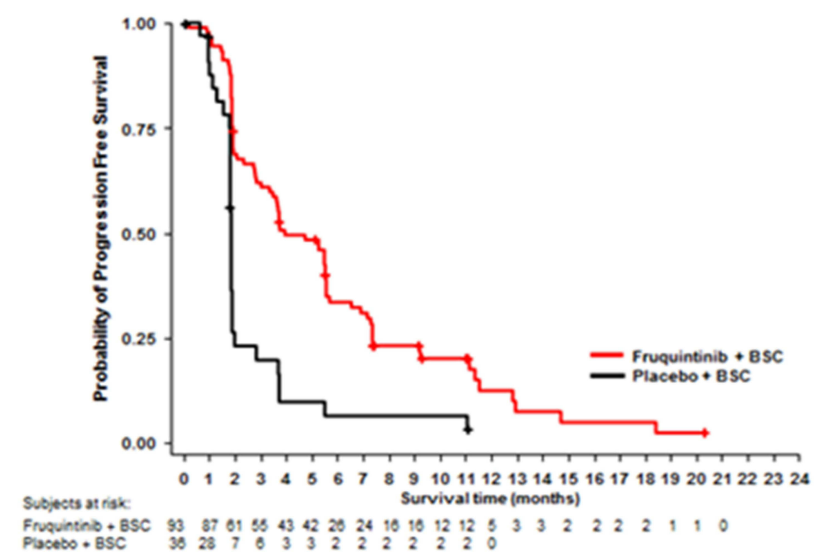

\section{Fruquintinib + \\ $\operatorname{BSC}(\mathrm{N}=93)$}

3.94

$(3.35,5.55)$
Placebo +

$\operatorname{BSC}(\mathrm{N}=36)$

1.84

$(1.81,1.87)$
$0.34(0.22,0.53)$

$<.001$

Figure 2 Comparison of progression-free survival in $m C R C$ patients with liver metastasis (A) and without liver metastasis (B). Abbreviations: BSC, best supportive care; HR, hazard ratio; $N$, total number patients.

$2(3 / 278=1.1 \%)$ and were mainly associated with underlying LM. Hepatic laboratory abnormalities by patients with and without liver metastasis are summarized in Table 3. The frequency of the adverse events of significant interest with hepatic laboratory abnormalities such as elevations in bilirubin, ALT, or AST was low. In both the fruquintinib and the placebo groups, more hepatic laboratory abnormalities were reported in patients with LM compared to those without metastasis. One patient in the fruquintinib group met Hy's law laboratory

Table 2 Response Rate in mCRC Patients with and without Liver Metastasis

\begin{tabular}{|c|c|c|c|c|}
\hline & \multicolumn{2}{|c|}{ Patients with Liver Metastasis } & \multicolumn{2}{|c|}{ Patients without Liver Metastasis } \\
\hline & $\begin{array}{l}\text { Fruquintinib+BSC } \\
\qquad(\mathrm{N}=185)\end{array}$ & $\begin{array}{l}\text { Placebo+ BSC } \\
\qquad(\mathrm{N}=102)\end{array}$ & $\begin{array}{l}\text { Fruquintinib+BSC } \\
\qquad(\mathrm{N}=93)\end{array}$ & $\begin{array}{c}\text { Placebo+BSC } \\
\qquad(\mathrm{N}=36)\end{array}$ \\
\hline Best overall response, $\mathrm{n}(\%)$ & & & & \\
\hline Complete response & 0 & 0 & $\mathrm{I}(\mathrm{I} . \mathrm{I})$ & 0 \\
\hline Partial response & $9(4.9)$ & 0 & $3(3.2)$ & 0 \\
\hline Stable disease & $106(57.3)$ & $9(8.8)$ & $54(58.1)$ & $8(22.2)$ \\
\hline Progressive disease & 59 (31.9) & $77(75.5)$ & $28(30.1)$ & $21(58.3)$ \\
\hline Not assessable & II (5.9) & $16(15.7)$ & $7(7.5)$ & $7(19.4)$ \\
\hline ORR, n (\%) & $9(4.9)^{*}$ & 0 & $4(4.3)$ & 0 \\
\hline DCR, n (\%) & $115(62.2)^{* *}$ & $9(8.8)$ & $58(62.4)^{* *}$ & $8(22.2)$ \\
\hline Median DOS, months $(95 \% \mathrm{Cl})$ & $5.5(4.8,5.5)$ & $3.7(3.1,4.8)$ & $5.7(5.5,7.4)$ & $3.7(2.8,11.0)$ \\
\hline
\end{tabular}

Notes: $* P<0.05, * * P<0.001, P$-value (fruquintinib vs placebo) based on fisher's exact test.

Abbreviations: BSC, best supportive care; $\mathrm{Cl}$, confidence interval; DCR, disease control rate; DOS, duration of stable disease; ORR, overall response rate. 


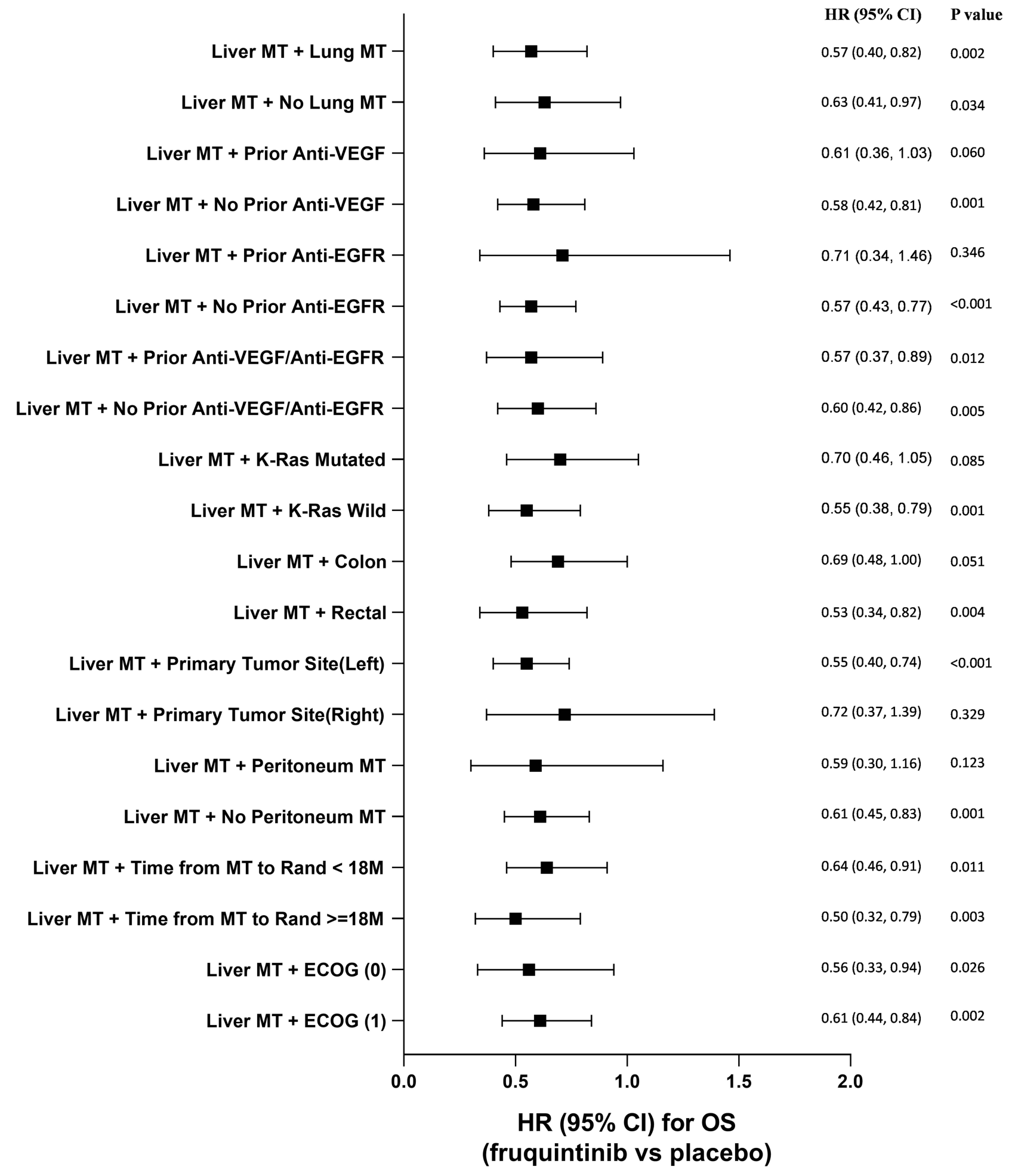

Figure 3 Comparison of overall survival for subgroup analysis of CRLM patients.

Abbreviations: $\mathrm{Cl}$, confidence interval; EGFR, epidermal growth factor receptor; HR, hazard ratio; M, months; MT, metastasis; VEGF, vascular endothelial growth factor. 


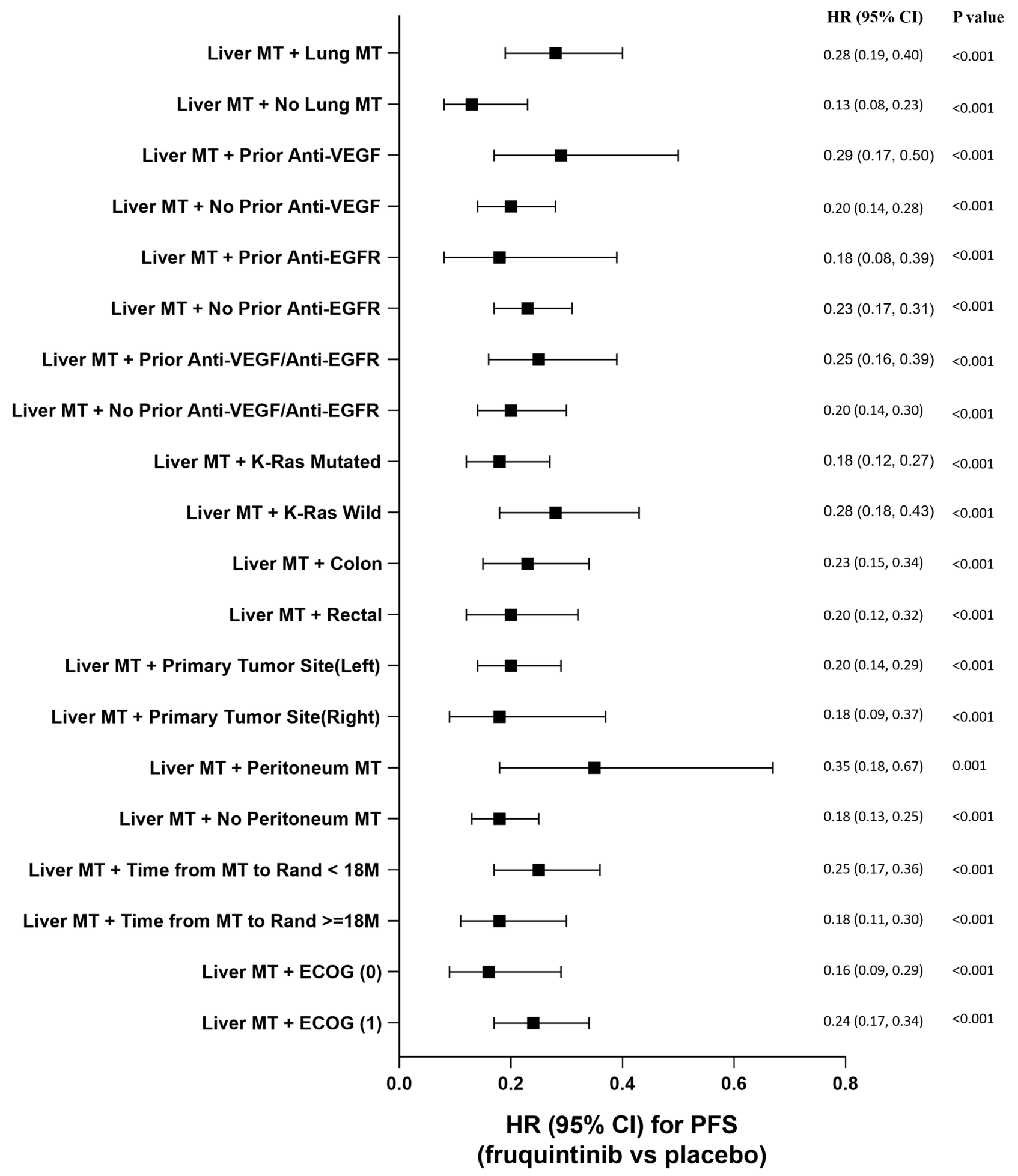

Figure 4 Comparison of progression free survival for subgroup analysis of CRLM patients.

Abbreviations: $\mathrm{Cl}$, confidence interval; EGFR, epidermal growth factor receptor; HR, hazard ratio; M, months; MT, metastasis; VEGF, vascular endothelial growth factor. 
Table 3 Treatment-Emergent Hepatotoxicity Events and Hepatic Laboratory Abnormalities in mCRC Patients with and without Liver Metastasis

\begin{tabular}{|c|c|c|c|c|}
\hline & \multicolumn{2}{|c|}{ Patients with Liver Metastasis } & \multicolumn{2}{|c|}{ Patients without Liver Metastasis } \\
\hline & $\begin{array}{l}\text { Fruquintinib+BSC } \\
\qquad \begin{array}{c}(\mathrm{N}=185) \\
n(\%)\end{array}\end{array}$ & $\begin{array}{c}\text { Placebo+BSC } \\
\qquad \begin{array}{c}(\mathbf{N}=102) \\
n(\%)\end{array}\end{array}$ & $\begin{array}{l}\text { Fruquintinib+BSC } \\
\qquad \begin{array}{c}(\mathbf{N}=93) \\
n(\%)\end{array}\end{array}$ & $\begin{array}{c}\text { Placebo+BSC } \\
\qquad \begin{array}{c}(\mathbf{N}=35) \\
n(\%)\end{array}\end{array}$ \\
\hline \multicolumn{5}{|c|}{ Treatment-emergent hepatotoxicity events } \\
\hline Any Grade & $7(3.8)$ & $2(2.0)$ & $2(2.2)$ & 0 \\
\hline Grade I & $5(2.7)$ & I ( $(1.0)$ & 0 & 0 \\
\hline Grade 2 & $2(1.1)$ & 0 & $\mathrm{I}(\mathrm{I} . \mathrm{I})$ & 0 \\
\hline Grade 3 & 0 & $\mathrm{I}(\mathrm{I} .0)$ & $\mathrm{I}(\mathrm{I} . \mathrm{I})$ & 0 \\
\hline \multicolumn{5}{|l|}{ Hepatic laboratory abnormalities } \\
\hline AST/ALT $>3 \times$ ULN and $\leq 5 \times$ ULN & $18(9.7)$ & $5(4.9)$ & $\mathrm{I}(\mathrm{I} . \mathrm{I})$ & I (2.9) \\
\hline AST/ALT >5x ULN & $10(5.4)$ & $3(2.9)$ & $2(2.2)$ & 0 \\
\hline Total bilirubin $>2 x$ ULN & $30(16.2)$ & $10(9.8)$ & $\mathrm{I}(\mathrm{I} . \mathrm{I})$ & I (2.9) \\
\hline $\begin{array}{l}\text { AST/ALT }>3 x \text { ULN and total bilirubin } \\
>2 x \text { ULN }\end{array}$ & $14(7.6)$ & I ( $(1.0)$ & 0 & I (2.9) \\
\hline Hy's law laboratory criteria* & $\mathrm{I}(0.5)$ & 0 & 0 & 0 \\
\hline
\end{tabular}

Notes: Data presented are from safety population. *AST/ALT $>3 x$ ULN, total bilirubin $>2 x$ ULN and ALP $<2 x$ ULN.

Abbreviations: ALP, alkaline phosphatase; ALT, alanine aminotransferase; AST, aspartate aminotransferase; BSC, best supportive care; ULN, upper limit of normal; N, total number patients; $n$, number of patients in each category.

criteria. This patient had underlying liver metastasis at baseline and was recovered after treatment thus was not considered as Hy's law case. No severe or fatal treatment-related hepatotoxicity was observed in the FRESCO trial.

\section{Discussion}

To the best of our knowledge, this is the first subgroup analysis by LM comparing efficacy and hepatotoxicity profile of fruquintinib versus placebo in Chinese patients with metastatic CRC who were not suitable for surgery and were receiving third-line or posterior-line therapies. Generally, mCRC patients with LM had worse survival than patients without LM, which was also observed as shortened OS in our study (5.98 vs 9.13 months, respectively, of placebo arm, Table 3). ${ }^{21,22}$ In the present CRLM subgroup analysis, median OS was significantly prolonged with fruquintinib compared with placebo ( $\mathrm{HR}=0.59,95 \%$ CI: 0.45-0.77). Also, the incremental median PFS for CRLM patients in the fruquintinib group was significantly higher than in the placebo group ( $\mathrm{HR}=0.22,95 \% \mathrm{CI}$ : 0.17 0.30). Overall, fruquintinib demonstrated significant survival benefit to the patients with CRLM. The efficacy results of this subgroup analysis are consistent with previously reported FRESCO study results. ${ }^{20}$ Furthermore, fruquintinib demonstrated a significant survival improvement compared to placebo in mCRC patients with both liver and lung metastases, and significantly reduced the mortality risk by $43 \%$. Besides liver metastasis, for mCRC patients, some other clinical and pathological factors might impact on prognosis, such as lung metastasis and K-RAS mutation. ${ }^{21,23}$ To confirm the efficacy of fruquintinib for CRLM patients with these factors, such as with prior antiVEGF/EGFR treatment, primary tumor site or K-RAS mutation, etc., we further analyzed the subgroups among CRLM patients. Survival benefits were shown to be consistent across subgroups regardless of prior anti-VEGF/ EGFR treatment, primary tumor site or K-RAS status. The results indicate that fruquintinib is effective in CRLM patients and significantly improves the OS and PFS for this population.

In the present analysis, fruquintinib showed clinically meaningful benefits in patients undergoing third-line or posterior-line therapies for patients with CRLM without observed accumulative hepatotoxicity. Overall, these subgroup analysis results are consistent with previously reported FRESCO intent-to-treatment population results. $^{20}$ In patients with CRLM, treatment-emergent hepatotoxicity of any grade occurred in $3.8 \%$ of patients in the fruquintinib group versus $2.0 \%$ of patients in the 
placebo group. None of the patients treated with fruquintinib had severe or fatal treatment-related hepatotoxicity. In safety population, no drug-induced liver injury was reported. The hepatotoxicity of fruquintinib was comparable with placebo in patients with CRLM.

There are some limitations to the present subgroup analysis. The present analysis was performed on a limited sample size, which reduces the power of a study/statistical test to find true differences. Also, similarity in baseline characteristics is not guaranteed within each subgroup. Thus, subgroup findings are not confirmatory and need further justification with more data.

\section{Conclusions}

As the first subgroup analysis of the efficacy and hepatic safety data of fruquintinib in Chinese patients with CRLM who were receiving third-line or posterior-line therapies, fruquintinib significantly lowered the death and recurrence risks for these patients. Notably, the treatment-emergent hepatotoxicity events rate was similar in CRLM patients compared to the placebo group. Also, survival benefits from fruquintinib were consistent across subgroups regardless of lung metastasis or prior target treatment in patients with CRLM in FRESCO.

\section{Data Sharing Statement}

The datasets generated during and/or analyzed during the current study are available on reasonable request. Data will be provided in a secure data sharing environment. Requests can be submitted through www.vivli.org.

\section{Ethics Approval and Informed Consent}

The study was approved by the institutional review board of the ethics committee from each study center: Fudan University Shanghai Cancer Center; 81 Hospital of PLA; Sun Yat-sen University Cancer Center; Harbin Medical University Cancer Hospital; The Sixth Affiliated Hospital of Sun Yat-sen University; Nantong Cancer Hospital; Zhejiang Cancer Hospital; The Second Hospital of Anhui Medical University; Sir Run Run Shaw Hospital, School of Medicine, Zhejiang University; Jiangsu Provincial Hospital; The Second Affiliated Hospital of Zhejiang University School of Medicine; Peking Union Medical College Hospital; First Affiliated Hospital Zhejiang University College of Medicine; ZhongShan Hospital Fudan University; 307 Hospital of PLA; Beijing Cancer
Center; Guangdong General Hospital; The First People's Hospital of Changzhou; Jilin Province Cancer Hospital; Shanghai First People's Hospital; The First Hospital of Jilin University; Xuzhou Central Hospital; The Affiliated Hospital of Medical College Qingdao University; The third Xiangya Hospital of Central South University; Liuzhou worker's hospital; Shanghai Changzheng Hospital; Beijing university ShenZhen hospital; and The Affiliated Hospital of Xuzhou Medical College. The study was conducted in accordance with the principles laid down by the Helsinki, 1964, its subsequent amendments, the Good Clinical Practice Guidelines, and laws and regulations in China regarding patient protection. All participants provided written informed consent for both their participation in this study and for its publication.

\section{Acknowledgments}

This study was funded and supported by Eli Lilly and Company and Hutchison MediPharma Limited. The authors thank Dr. Shu Wang (Eli Lilly and Company, China) for her contribution to the scientific publication editing the first draft and the final revision. The authors would like to thank Dr. Rakesh Ojha, PhD, and Deepika Kajarekar from Syneos Health for medical writing support, and Angela C. Lorio, ELS, from Syneos Health for editorial support in the preparation of this manuscript.

\section{Author Contributions}

All authors: (a) made substantial contributions to the conception or design of the work; or the acquisition, analysis, or interpretation of data for the work; AND (b) drafted the work or revised it critically for important intellectual content; AND (c) agreed on the journal to which the article will be submitted, gave final approval of the version to be published; (d) agreed to be accountable for all aspects of the work in ensuring that questions related to the accuracy or integrity of any part of the work are appropriately investigated and resolved.

\section{Funding}

The FRESCO trial was co-funded by Eli Lilly and Company (Indianapolis, IN, USA) and Hutchison MediPharma (Shanghai, China). The role of the funding source includes study design, data acquisition, analysis and interpretation of data, and the decision to submit the article for publication. All authors had full access to all of the data in this study and take complete responsibility for 
the integrity of the data and accuracy of the data analysis. Medical writing assistance support for this publication was funded by Eli Lilly and Company.

\section{Disclosure}

Ning Wang, Bin Zhang, and Qiang Zhang are employees of Eli Lilly and Company. Weiguo Su and Xiaojun Guo are employees of Hutchison MediPharma Limited. Dr Lin Shen reports non-financial support from Lilly, non-financial support from HMPL, during the conduct of the study; grants from Beijing Xiantong Biomedical Technology, grants from Qilu Pharmaceutical, grants from ZaiLab Pharmaceutical, grants from Beihai Kangcheng (Beijing) Medical Technology, grants from Jacobio Pharmaceuticals; received consulting fees from MSD, Merck, Mingji Biopharmaceutical, Haichuang pharmaceutical, Harbour Biomed, and BI; also received fees for speakers Bureaus from Hutchison Whampoa, Hengrui, ZaiLab, CSTONE pharmaceutical; and a member of Advisory Board of Rongchang Pharmaceutical, ZaiLab, CSTONE Pharmaceutical, BMS, outside the submitted work. The other authors have nothing to disclose.

\section{References}

1. Rawla P, Sunkara T, Barsouk A. Epidemiology of colorectal cancer: incidence, mortality, survival, and risk factors. Prz Gastroenterol. 2019;14(2):89-103. doi:10.5114/pg.2018.81072

2. Haggar FA, Boushey RP. Colorectal cancer epidemiology: incidence, mortality, survival, and risk factors. Clin Colon Rectal Surg. 2009;22 (4):191-197. doi:10.1055/s-0029-1242458

3. Chen W, Zheng R, Baade PD, et al. Cancer statistics in China, 2015. CA Cancer J Clin. 2016;66(2):115-132. doi:10.3322/caac.21338

4. Pourhoseingholi MA. Increased burden of colorectal cancer in Asia. World J Gastrointest Oncol. 2012;4(4):68-70. doi:10.4251/wjgo.v4. i4.68

5. Van de Velde CJH. Treatment of liver metastases of colorectal cancer. Ann Oncol. 2005;16(Suppl 2):ii144-ii149. doi:10.1093/annonc/ $\operatorname{mdi} 702$

6. Welch JP, Donaldson GA. The clinical correlation of an autopsy study of recurrent colorectal cancer. Ann Surg. 1979;189(4):496-502. doi:10.1097/00000658-197904000-00027

7. Xu R, Wang W, Zhu B, et al. Disease characteristics and treatment patterns of Chinese patients with colorectal cancer: a retrospective study using medical records from China. BMC Cancer. 2020;131 (20). doi:10.1186/s12885-020-6557-5

8. Chow FC, Chok KS. Colorectal liver metastases: an update on multidisciplinary approach. World J Hepatol. 2019;11(2):150-172. doi:10.4254/wjh.v11.i2.150
9. Blackham AU, Swett K, Levine EA, Shen P. Surgical management of colorectal cancer metastases to the liver: multimodality approach and a single institutional experience. Colorectal Cancer. 2013;2 (1):73-88. doi: $10.2217 /$ crc. 12.80

10. Xu F, Tang B, Jin TQ, Dai CL. Current status of surgical treatment of colorectal liver metastases. World J Clin Cases. 2018;6(14):716-734. doi:10.12998/wjcc.v6.i14.716

11. Aparicio J, Fernandez-Martos C, Vincent JM, et al. FOLFOX alternated with FOLFIRI as first-line chemotherapy for metastatic colorectal cancer. Clin Colorectal Cancer. 2005;5(4):263-267. doi:10.3816/CCC.2005.n.037

12. Jonker D, Rumble RB, Maroun J; Gastrointestinal Cancer Disease Site Group of Cancer Care Ontario's Program in Evidence-Based Care. Role of oxaliplatin combined with 5-fluorouracil and folinic acid in the first- and second-line treatment of advanced colorectal cancer. Curr Oncol. 2006;13(5):173-184. doi:10.3747/co.v13i5.99

13. Limaiem F, Bouraoui S. Chemotherapy-induced liver injury in metastatic colorectal cancer: about 48 cases. Pan Afr Med J. 2018;30:198. doi:10.11604/pamj.2018.30.198.15548

14. Maor Y, Malnick S. Liver injury induced by anticancer chemotherapy and radiation therapy. Int $J$ Hepatol. 2013;2013:815105. doi:10.1155/ 2013/815105

15. Choti MA. Chemotherapy-associated hepatotoxicity: do we need to be concerned? Ann Surg Oncol. 2009;16(9):2391-2394. doi:10.1245/ s10434-009-0512-7

16. Chen D, Gu K, Wang H. Optimizing sequential treatment with anti-EGFR and VEGF mAb in metastatic colorectal cancer: current results and controversies. Cancer Manag Res. 2019;11:1705-1716. doi:10.2147/CMAR.S196170

17. Schirripa M, Lenz HJ. Colorectal cancer: overcoming resistance to anti-EGFR therapy-where do we stand? Nat Rev Gastroenterol Hepatol. 2016;13(5):258-259. doi:10.1038/nrgastro.2016.52

18. De Mattia E, Cecchin E, Toffoli G. Pharmacogenomics of intrinsic and acquired pharmacoresistance in colorectal cancer: toward targeted personalized therapy. Drug Resist Updat. 2015;20:39-70.

19. Zhang Y, Zou JY, Wang Z, Wang Y. Fruquintinib: a novel antivascular endothelial growth factor receptor tyrosine kinase inhibitor for the treatment of metastatic colorectal cancer. Cancer Manag Res. 2019;11:7787-7803. doi:10.2147/CMAR.S215533

20. Li J, Qin S, Xu RH, et al. Effect of fruquintinib vs placebo on overall survival in patients with previously treated metastatic colorectal cancer: the FRESCO randomized clinical trial. JAMA. 2018;319 (24):2486-2496. doi:10.1001/jama.2018.7855

21. V T Broadbridge, C S Karapetis, C Beeke et al. Do Metastatic Colorectal Cancer Patients Who Present With Late Relapse After Curative Surgery Have a Better Survival?. Br J Cancer. 2013;109 (5):1138-43.

22. Kanas G, Aliki T, Primrose J et al. Survival after liver resection in metastatic colorectal cancer: review and meta-analysis of prognostic factors. Clin Epidemiol. 2012;4:283-301.

23. F. Jones, D. Edelstein, K. Wichner, C. Ross, F. Holtrup. 2012 Concordance of RAS mutation status in metastatic CRC patients bycomparison of results from circulating tumor DNA and tissuebased RAS testing. European Journal of Cancer. 2015;51(3): S331-S332. 


\section{Publish your work in this journal}

OncoTargets and Therapy is an international, peer-reviewed, open access journal focusing on the pathological basis of all cancers, potential targets for therapy and treatment protocols employed to improve the management of cancer patients. The journal also focuses on the impact of management programs and new therapeutic agents and protocols on patient perspectives such as quality of life, adherence and satisfaction. The manuscript management system is completely online and includes a very quick and fair peer-review system, which is all easy to use. Visit http://www.dovepress.com/ testimonials.php to read real quotes from published authors.

Submit your manuscript here: https://www.dovepress.com/oncotargets-and-therapy-journal 